\title{
Akathisia in Elderly Female Patient with Schizophrenia upon concurrent use of Risperidone and Sertraline
}

\author{
Jaskirat Singh Sidhu1, Austin Cusick ${ }^{2}$, Shehla Yasin Belgam Syed ${ }^{3}$, Pankaj Bansal ${ }^{4}$, Amandeep $^{1}$ \\ Goyal $^{5 *}$ \\ ${ }^{1}$ PGY-3 Psychiatry Resident, Center for Behavioral Medicine, UMKC School of Medicine, USA \\ ${ }^{2}$ Medical Student, Ohio University Heritage College of Osteopathic Medicine, USA \\ ${ }^{3}$ Boston University, Boston MA, USA
}

${ }^{4}$ Internal Medicine/Rheumatology Attending, Mayo Clinic Health System, USA

${ }^{5}$ Internal Medicine Attending, Clinical Preceptor, Marietta Memorial Hospital, USA

*Corresponding author: Amandeep Goyal, Internal Medicine Attending, Clinical Preceptor, Marietta Memorial Hospital, 401 Matthew St. Marietta, Ohio, USA

\section{ARTICLE INFO}

Received: 幽 April 17, 2020

Published: 幽 April 23, 2020

Citation: Jaskirat Singh S, Austin C, Shehla Y B S, Pankaj B, Amandeep G. Akathisia in Elderly Female Patient with Schizophrenia upon concurrent use of Risperidone and Sertraline. Biomed J Sci \& Tech Res 27(2)2020. BJSTR. MS.ID.004469.

Keywords: Akathisia; Risperidone; Sertraline; Extra-pyramidal symptoms

Abbreviations: SGA: Second Generation Antipsychotics; EPS: Extrapyramidal Symptoms; FGA: First Generation Antipsychotics; SSRI: Selective Serotonin Reuptake Inhibitors; TD: Tardive Dyskinesia; UTI: Urinary Tract Infection

\section{ABSTRACT}

Second Generation Antipsychotics (SGAs) are used in the treatment of various psychiatric disorders. While SGAs have a risk of side effects including Extrapyramidal Symptoms (EPS), they are generally considered to have a lower risk than their counterparts First Generation Antipsychotics (FGA's). In the spectrum of SGAs, risperidone has the highest risk and clozapine has the lowest risk for causing EPS. Several other characteristics and steps of care may influence the development of EPS including advanced age, female sex, non-caucasian race, history of EPS, higher doses of antipsychotics, choice of antipsychotics, and presence of comorbid medical conditions. Selective Serotonin Reuptake Inhibitors (SSRIs) are also known to cause EPS, although the risk is low with sertraline having the lowest propensity amongst SSRIs. We present a case of 57-year old Hispanic female with schizophrenia who had responded favorably to $6 \mathrm{mg}$ of risperidone without any side-effects but then developed akathisia on $8 \mathrm{mg}$ of risperidone and $25 \mathrm{mg}$ of Sertraline. This case corresponds to the current notion about the various risk factors that predispose a patient to developing EPS while using risperidone. The complicated care management of this patient with FGA's and her gender with advanced age all may have increased her propensity to develop EPS. Co-administration of risperidone with sertraline provided enough additive effect, in combination with underlying risk factors, to induce EPS in this patient.

\section{Introduction}

Second Generation Antipsychotics (SGAs) were introduced into the market in the 1980s. Among SGAs, risperidone has the highest risk and clozapine has the lowest risk for causing Extrapyramidal Symptoms (EPS). Risk factors for the development of EPS include advanced age, female sex, non-Caucasian race, history of EPS, higher doses of antipsychotics, choice of antipsychotics, and presence of comorbid medical conditions [1]. EPS can include symptoms of dystonia, akathisia, tardive dyskinesia, and parkinsonism. Akathisia is a movement disorder where the patient has a subjective feeling of restlessness with an irresistible urge to move, objectively viewed by providers as repetitive movements. Prevalence of akathisia on SGA monotherapy is $10.9 \%$ [2]. Reviews of literature revealed a few case studies reporting EPS from risperidone at doses higher than $6 \mathrm{mg}$. The North American Trial depicted that risperidone at the doses of $10 \mathrm{mg}$ and above had side effect profiles like haloperidol, and at doses more than $6 \mathrm{mg}$, the need for anticholinergic treatment was higher [3]. 
There is also one case study where a patient had a good response to $20 \mathrm{mg}$ of risperidone without any side-effects contrary to the current literature [4], and another prospective study depicted contrasting results that the prevalence of tardive dyskinesia (TD) with risperidone is similar to that of olanzapine [5]. Selective Serotonin Reuptake Inhibitors (SSRIs) are also known to cause EPS, although the risk is low. There have been a few studies and reports showing that SSRIs increase the risk of EPS especially when used concomitantly with antipsychotics in patients with additional risk factors [6,7]. Among SSRIs, fluoxetine has the highest propensity to cause EPS, and sertraline has the lowest risk. We present a case of 57-year old Hispanic female with schizophrenia who had responded favorably to $6 \mathrm{mg}$ of risperidone without any sideeffects but then developed akathisia on $8 \mathrm{mg}$ of risperidone and 25 mg of Sertraline. This case corresponds to the current notion about the various risk factors that predispose a patient to developing EPS while using risperidone.

\section{Case Report}

A 57-year-old Hispanic female with a past psychiatric history of Schizophrenia and Nicotine use disorder, was admitted to the inpatient geriatric psychiatry unit for worsening of psychosis with suicidal ideations plus a plan to jump off a balcony. Patient past medical history includes hyperthyroidism and acute Urinary Tract Infection (UTI). Medically, she received antibiotic treatment for her UTI and Methimazole $10 \mathrm{mg}$ by mouth (PO) three times a day (TID) was started for hyperthyroidism. On the first day of admission, the patient was prescribed Haloperidol $2.5 \mathrm{mg}$ by mouth twice a day (BID) for psychosis, and it was titrated up to $10 \mathrm{mg}$ by mouth at night (QHS) on day 14. The thinking behind its use was a quick onset of sedative effects, easy financial accessibility, and lesser risk of anticholinergic effects than of other first-generation antipsychotics given her acute UTI. Mirtazapine $7.5 \mathrm{mg}$ PO QHS was added for insomnia and appetite stimulation on day 14 and increased to 15 mg PO QHS on day 22. On day 6, Lorazepam $0.5 \mathrm{mg}$ PO BID was added, and slowly titrated to $1 \mathrm{mg}$ PO QID on day 20, for possible catatonia

On day 14, Diphenhydramine 50mg PO QHS was added for possible EPS from Haloperidol. On day 21, Haloperidol was discontinued due to ineffectiveness and development of EPS. We also discontinued Diphenhydramine and Lorazepam as they were given for EPS and catatonia respectively. On day 21, we added Risperdal-M tab 2mg PO BID for psychosis and added as needed Olanzapine and Quetiapine for anxiety/agitation. On day 20, we added Sertraline $25 \mathrm{mg}$ PO daily for depressed mood. This was discontinued on day 27 as her symptoms were more consistent with schizophrenia rather than depressive disorder. On day 23, we increased Risperdal-M Tab to 3mg PO BID and then to $4 \mathrm{mg}$ PO BID on day 28. While on risperidone, the patient showed only slight improvement and developed akathisia. Consequently, we stopped Risperidone on day 30 due to the development of akathisia and poor response. We then began Olanzapine 2.5mg PO BID for psychosis and Propranolol 10mg PO BID for akathisia, and Mirtazapine was continued. Akathisia improved with 1 week of stopping the risperidone and on day 37 Propranolol was stopped. Patient did not develop akathisia or EPS after stopping Risperidone and while on olanzapine.

\section{Discussion}

Risperidone is Second Generation Antipsychotic (SGA) from the benzisoxazole group, with serotonin 5-HT2A and dopamine D2 antagonism properties. SGAs bind loosely to D2 dopamine receptors compared to FGAs, and thus have a lower risk in causing EPS. Various hypotheses have been proposed to explain the higher propensity of risperidone to cause EPS despite being an SGA. Risperidone is a potent antagonist of D2 receptors, and this dopamine blockade increases acetylcholine and subsequently EPS. Positron Emission Tomography based studies have demonstrated that adequate dopamine 2 (D2) antagonism occurs at doses of up to $4 \mathrm{mg}$ per day leading to the therapeutic effects. Dosages above $6 \mathrm{mg}$ /day are of a higher incidence of EPS. SSRIs increase serotonin that has antagonizing effects on dopamine. When SSRIs are administered with antipsychotics, it further increases the risk of EPS development. Another school of thought suggests that prolonged antipsychotic medication use results in increased postsynaptic dopamine hypersensitivity and damage to the striatal cholinergic interneurons resulting in amplified risk in developing EPS [8]. This is again consistent with our clinical case. The patient had a chronic history of schizophrenia and has been trialed on various medications in the past. Additionally, as Woerner et al. [5], had suggested that previous exposure to the FGAs (especially Haloperidol) is a risk factor for the development of EPS again consistent to our patient, who was trialed on Haloperidol before switching her to Risperidone.

\section{Conclusion}

In sum, there have been numerous reported clinical cases in the past demonstrating dose related EPS from Risperidone, and as mentioned in the introduction there have also been some studies showing contrasting results. This clinical case highlights the significance of assessing the above-mentioned risk factors before prescribing higher doses of risperidone, gradually titrating the dose of risperidone, and exerting caution in concomitant use of SSRI's with antipsychotics especially in high-risk patients. This scrutiny can avoid many of the adverse effects to our patients, which can improve medication adherence which is a major concern in this patient population.

\section{Conflicts of Interest}

The authors have no conflicts or interests to declare.

\section{Disclosures}

Authors have nothing to disclose. 


\section{References}

1. Thomson SR, Chogtu B, Bhattacharjee D, Agarwal S (2017) Extrapyramidal Symptoms Probably Related to Risperidone Treatment: A Case Series. Ann Neurosci 24(3): 155-163.

2. Berna F, Misdrahi D, Boyer L, Aouizerate B, Brunel L, et al. (2015) FACESZ (FondaMental Academic Centers of Expertise for Schizophrenia) group. Akathisia: prevalence and risk factors in a community-dwelling sample of patients with schizophrenia. Results from the FACE-SZ dataset. Schizophr Res 169(1-3): 255-261.

3. Marder SR, Davis JM, Chouinard G (1997) The effects of risperidone on the five dimensions of schizophrenia derived by factor analysis: combined results of the North American trials. J Clin Psychiatry 58(12): 538-546.

4. Ray A, Mukherjee B (2013) A case of resistant schizophrenia responding at a higher than recommended dose of risperidone without significant side effects. Indian J Pharmacol 45(1): 100-101.

ISSN: 2574-1241

DOI: 10.26717/BJSTR.2020.27.004469

Amandeep Goyal. Biomed J Sci \& Tech Res

(C) This work is licensed under Creative

Submission Link: https://biomedres.us/submit-manuscript.php
5. Woerner MG, Correll CU, Alvir JM, Greenwald B, Delman H, et al. (2011) Incidence of tardive dyskinesia with risperidone or olanzapine in the elderly: results from a 2-year, prospective study in antipsychotic-naïve patients. Neuropsychopharmacology 36(8): 1738-1746.

6. Lane RM (1998) SSRI-induced extrapyramidal side-effects and akathisia: implications for treatment. J Psychopharmacol 12(2): 192-214.

7. Schillevoort I, van Puijenbroek EP, de Boer A, Roos RA, Jansen PA, et al. (2002) Extrapyramidal syndromes associated with selective serotonin reuptake inhibitors: a case-control study using spontaneous reports. Int Clin Psychopharmacol 17(2): 75-79.

8. Bobes J, Rejas J, Garcia Garcia M, Rico Villademoros F, García Portilla MP, et al. (2002) Frequency of Extrapyramidal Adverse Reactions in Schizophrenic Outpatients Treated with Risperidone, Olanzapine, Quetiapine or Haloperidol: Results of the EIRE Study. Clin Drug Investig 22(9): 609-622.

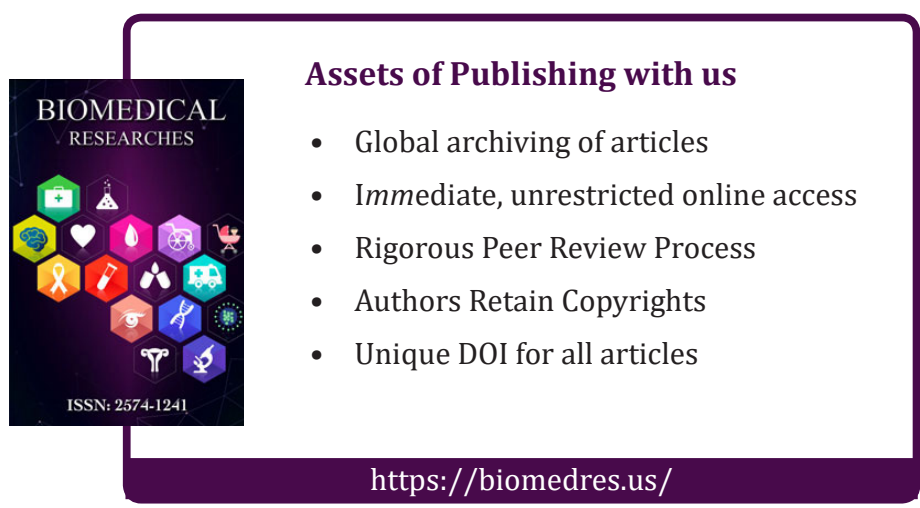

